\title{
Sonographic Cervical Volumetry in Higher Order Multiple Gestation
}

\author{
Alexander Strauss Ivo Heer Susanne Fuchshuber Udo Janssen \\ Peter Hillemanns Hermann Hepp \\ Department of Obstetrics and Gynecology - Grosshadern, University of Munich, Munich, Germany
}

\section{Key Words}

Cervical volume $\cdot$ Cervical length $\cdot$ Higher order multiple gestation · 3D-ultrasound - Cervical incompetence .

Preterm birth dominal 2D-plane was obstructed. When cervical length was obtainable by a conventional scan, the technically more complex 3D-imaging did not provide further information.

Copyright $\left({ }^{2} 2001\right.$ S. Karger AG, Basel

\begin{abstract}
Objective: The aim of this study of multifetal pregnancies was the comparison of three-dimensional (3D) volumetry of the cervix, conventional sonographic cervical length measurement and clinical assessment. Methods: 10 mothers were investigated in an observational study between 5/1999 and 9/2000. A total of 34 consecutive 2Dand 3D-transabdominal ultrasound measurements were performed. Results: Volumetry of the cervix was possible in all 34 exams. 2D-cervical length assessment could not be obtained in $6 \%$ because the presenting fetal part obstructed the sonographic plane. Both methods allowed equal judgement of the configuration of the cervix. A significant correlation was found between mean 2D-cervical length (28.7 mm, 7.7 SD) and mean cervical volume $\left(30.0 \mathrm{~cm}^{3}, 16.0 \mathrm{SD}\right)$. Parity, subjective preterm labor or need of tocolytics showed no correlation with any biometrical parameter studied. Conclusion: Volumetry was superior for the assessment of cervical biometry and conformation in women when the transab-
\end{abstract}

\section{KARGER}

Fax + 41613061234

E-Mail karger@karger.ch

www. karger.com
(C) 2001 S. Karger AG, Basel

$1015-3837 / 01 / 0166-0346 \$ 17.50 / 0$

Accessible online at:

www.karger.com/journals/fdt

\section{Introduction}

Multiple births disproportionately contribute to perinatal mortality and morbidity. The incidence of preterm delivery is reported to be $66-100 \%$ in higher order multiple gestation and complications of prematurity have the highest impact on the outcome of these pregnancies [1]. The risk of preterm birth and analogous adverse fetal outcome is further known to increase with the number of fetuses [2]. The ability to predict the onset of preterm labor or monitor cervical incompetence is therefore of tremendous value.

The mechanisms leading to early second trimester cervical incompetence have yet to be fully understood. It is not established, whether cervical failure results from a lack of cervical support (passive changes), uterine contractions (active changes) or a combination of the two [3, 4]. The diagnosis of cervical incompetence is difficult for the lack of well-defined maternal symptoms. 'Painless 
dilatation' can either be palpated during second trimester or be visualized sonographically. If antepartum cervical assessment can better define the risk of premature delivery in multifetal gestation, it would be of great clinical value to establish a method that is simple, reproducible, generally applicable and cost effective. In order to evaluate the sonographic changes of conformation of the cervix, different authors [5, 6] developed prognostic quantifiable cervical scoring systems. Nevertheless, Bishop's pelvic score remains the most commonly applied measure of cervical ripening $[7,8]$.

Large prospective series have confirmed a close correlation of sonographic cervical length measurements obtained between 20-24 weeks of gestation and the risk of preterm birth. In a study of 2,915 pregnancies within a low-risk population, cervical length measurements of $<20 \mathrm{~mm}$ predicted preterm delivery before 35 weeks in $23 \%$. Similarly, another study showed a $48 \%$ positive predictive value for delivery prior to 32 weeks when cervical length measured $<15 \mathrm{~mm}$ before 28 weeks. The authors report specificity to be as high as $97 \%$ (95\% confidence interval of 96-98) [9, 10]. Thus ultrasound assessment of the cervix in second trimester may help to detect and prevent prematurity by unveiling cervical changes that predict cervical incompetence in later pregnancy. Currently, two-dimensional (2D) sonographic surveillance of cervical length is part of routine prenatal management in pregnancies at risk, e.g. preterm labor, PPROM (preterm premature rupture of the membranes), previous preterm delivery or higher order multiple gestation [4, 11, 12].

$3 \mathrm{D}$-ultrasound is the major recent technical progress in prenatal diagnosis. Accuracy of 3D-volume evaluation has been proven by different investigators measuring human kidney-, fetal lung-, ovarian follicle- or cervical cancer volumes, respectively [13-16]. The studies published so far, comparing cervical volume to conventional 2D-scans in pregnancy, failed to demonstrate the hypothetically expected advantage of 3D-ultrasound identifying women at an increased risk of preterm birth [17].

In contrast to most studies on ultrasonographic cervical length assessment, we performed our measurements transabdominally in order to avoid the cervical manipulation and potential induction of preterm birth. The transabdominal scan is a safe (no risk of promoting ascending vagino-cervical infection) and reproducible (no distortion of the cervical conformation through the transducer) method to assess the cervix uteri in an either $2 \mathrm{D}$ or $3 \mathrm{D}$ scan. On the other hand, overdistension of the maternal bladder cannot only induce abdominal discomfort but may also lead to an elongation of the cervix or could cause an open internal os to appear closed. In our opinion, noninvasive cervical assessment in higher order multiple pregnancies should not primarily intend a risk-prediction of preterm birth, as these pregnancies are generally delivered at an early gestational age. Rather, we intended to investigate consecutive measurements as means to monitor the continuous alteration of the cervix and, as a result, potentially modify prenatal management (e.g. bed rest, hospitalization, antibiotics, tocolysis).

The aim of our study was to evaluate whether 3Dultrasound of the cervix in a multiple pregnancy is an exact and practicable alternative either to conventional sonography or to palpation of the pelvis. Our hypothesis was that 3D-ultrasound may better predict cervical shortening than 2D gray scale sonography or Bishop's pelvic score. To our knowledge, this is the first report on cervical volumetry investigating cervical changes in higher order multiple gestation.

\section{Material and Methods}

10 patients pregnant with higher order multiples ( 9 triplet-, 1 quadruplet-pregnancies), managed at Grosshadern Hospital, Department of Obstetrics and Gynecology, Munich University between May, 1999 and September, 2000, were prospectively included in a study for assessment of cervical length as well as cervical volume. In all mothers, sequential transabdominal ultrasound measurements (34) were performed with Sequoia 512 (Acuson, Mountain View, Calif., USA) using a curved array probe (5.0 MHz). Volumetry was calculated using an external 3D position electronic system based on an orthogonal magnetic field system (FreeScan, 3D-EchoTech, Hallbergmoos, Germany). Each investigation consisted of a 2D-measurement of cervical length, assessment of the conformation of the internal and external os, as well as the dilatation of the cervical canal. In separate sagittal as well as transverse sweeps, a sonographic volume of the cervix uteri was scanned. 3D sonographic data was acquired, calculated and stored on a double Pentium II $400 \mathrm{MHz}$ personal computer. Analysis of cervical volume was completed later, timeindependent from acquisition (fig. 1). In the case of 'funneling' of the internal os or an opened cervical canal, the corresponding volume had to be subtracted from the total cervical volume. To avoid interobserver variance all scans were performed by the same investigator (A.S.). The frequency of antenatal visits was established individually, based on clinical criteria - weekly or biweekly. In addition to transabdominal ultrasound, manual pelvic examination was performed to evaluate cervical length and effacement, consistency and position as well as position of the presenting fetal part. Sonographic data was compared to the pelvic score established by Bishop [6-8]. Values were expressed as mean \pm SD. $\chi^{2}$ test, Mann-Whitney-U test and Pearson's correlation test were used for statistical analysis. Significance was defined at $\mathrm{p}<0.05$.

Fetal Diagn Ther 2001;16:346-353

Cervical Volumetry in Multiple Gestation 
Fig. 1. 3D-cervical volume assessment (triplet gestation, $22+4$ weeks of gestation, cervical length $34 \mathrm{~mm}$ (2D), cervical volume $\left.44.9 \mathrm{~cm}^{3}\right)$. Definition of the target volume in 4 planes enclosing an angle of $45^{\circ}$ each.

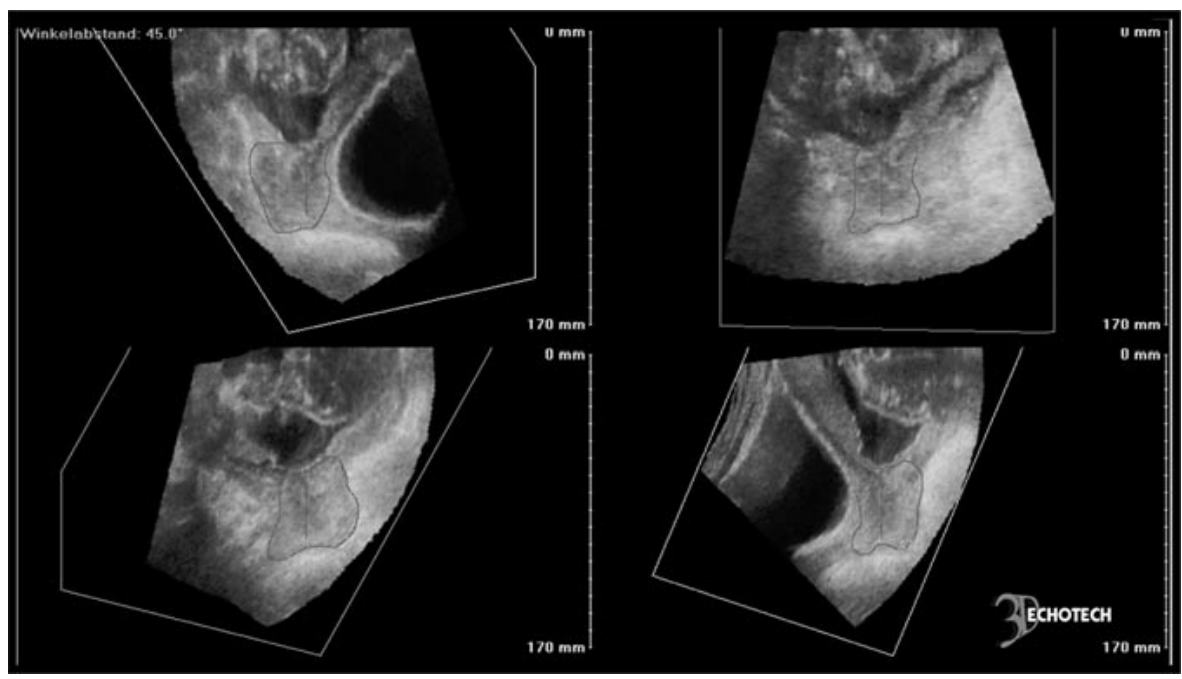

\section{Results}

In the study period (5/1999-9/2000) 9 triplet and 1 quadruplet mothers underwent repeated sonographic studies in order to characterize ongoing changes in cervical conformation. All higher order multiple pregnancies resulted from different techniques of modern reproductive medicine - IVF (60\%), ICSI (20\%), hormonal stimulation therapy (20\%). Mean maternal age was $32 \pm 3$ years and mean parity of mothers pregnant with higher order multiples was $1.4 \pm 1.0$. Maternal complications such as preterm labor, cervical incompetence, maternal physical decompensation or premature preterm rupture of membranes were present in $6,4,2$ or 2 pregnancies, respectively. In all mothers, preterm contractions were treated with magnesium sulfate per os. Intravenous tocolytics (Fenoterol) were used in $80 \%$ of studied pregnancies for a mean duration of $25 \pm 25$ days. None of the mothers carrying triplets had a history of surgical procedures of the cervix neither during nor before their current pregnancy. The quadruplet pregnancy, however, was treated with two cervical cerclage procedures, at an outside facility in early second trimester. Mean gestational age at the time of hospitalization was $176 \pm 24$ days. Following this preventive scheme withholding premature birth the mean interval to delivery was $39 \pm 18$ days. Mean gestational age at delivery was $215 \pm 19$ days corresponding to a mean birth weight of $1,380 \pm 406 \mathrm{~g}$. In all pregnancies a cesarean section was performed.

Cervical competence was monitored clinically through regular manual palpation of the pelvis. As established as the pelvic score by Bishop in 1964, cervical dilatation,

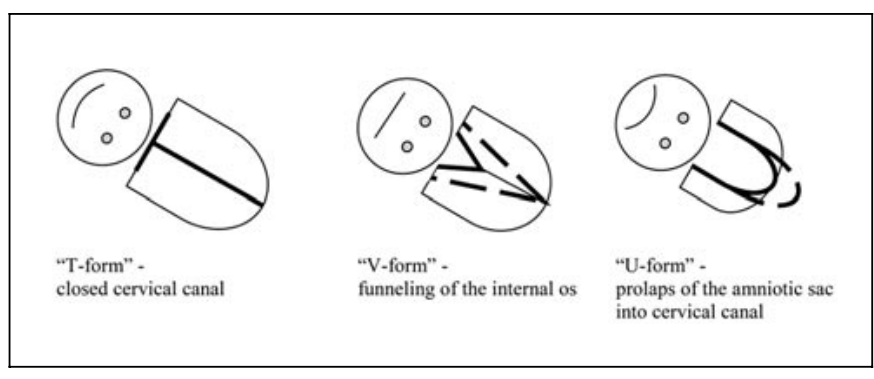

Fig. 2. Conformation changes of the internal os of cervix uteri. [Modified from 19.]

effacement, consistency and position as well as the station of the presenting fetal part were recorded (table 1) [7]. Mean pelvic score was $4 \pm 1.4$. Bishop's score did not correlate with preterm labor and/or the need for tocolysis: $3.4 \pm 1.9$ vs. $2.0 \pm 1.9$ in the absence of symptoms ( $p=$ $0.184)$.

\section{Sonography}

A mean of 3 (range 1-7) sonographic examinations of the cervix uteri were prospectively compared in all 10 multiple pregnancies. In order to optimize transabdominal access to the cervix, filled maternal urinary bladder was required. Volume calculations were obtainable in all investigations (34) whereas 2D-cervical length and configuration assessment failed in 6\%. In these cases, longitudinal scanning for length measurement was completely obstructed by the presenting part of the leading fetus. In the other mothers, the presenting part of the fetal body 
Fig. 3. Correlation between the direct cervical length measurements obtained via 2Dscans and those obtained through reconstruction from 3D-scans. Correlation coefficient: $r=0.75$.

Table 1. Bishop's pelvic scoring system (total score is the summation of the scores of the five criteria) [7]

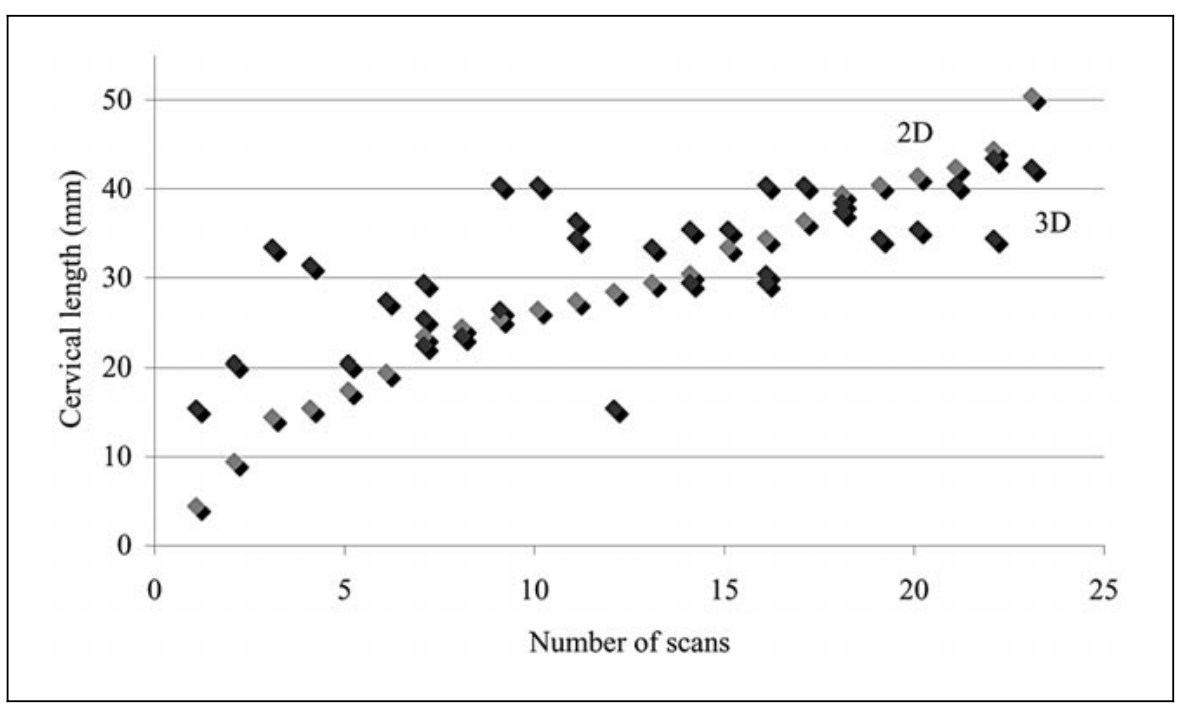

\begin{tabular}{lllll}
\hline Pelvic score & 0 & 1 & 2 & 3 \\
\hline Station of presenting part, cm & -3 & -2 & -1 & +1 \\
Cervical dilatation, cm & closed & $1-2$ & $3-4$ & $5+$ \\
Cervical effacement, $\%$ & $0-39$ & $40-59$ & $60-79$ & $80+$ \\
Cervical consistency & firm & medium & soft & \\
Cervical position & posterior & mid-position & anterior & \\
\hline
\end{tabular}

Table 2. Cervical morphology compared to $2 \mathrm{D}$ and $3 \mathrm{D}$ ultrasound biometry and duration of pregnancy (mean \pm SD)

\begin{tabular}{|c|c|c|c|c|c|c|c|c|c|}
\hline & \multicolumn{3}{|l|}{ Internal os } & \multicolumn{3}{|c|}{ Cervical canal } & \multicolumn{3}{|l|}{ External os } \\
\hline & funneling & no funneling & & open & closed & & open & closed & \\
\hline Volume, $\mathrm{cm}^{3}$ & $28.8 \pm 15.5$ & $26.5 \pm 8,4$ & NS & $30.9 \pm 18.7$ & $25.0 \pm 7.0$ & NS & $38.9 \pm 15.1$ & $26.7 \pm 10.8$ & NS \\
\hline Length, mm & $32.6 \pm 9.6$ & $27.3 \pm 4.5$ & NS & $28.6 \pm 9.3$ & $27.7 \pm 4.9$ & NS & $35.0 \pm 7.8$ & $27.8 \pm 6.6$ & NS \\
\hline $\begin{array}{l}\text { Duration of } \\
\quad \text { pregnancy, days }\end{array}$ & $26.2 \pm 15.2$ & $28.3 \pm 20.0$ & NS & $23.9 \pm 16.2$ & $27.4 \pm 18.5$ & NS & $29.3 \pm 22.8$ & $23.9 \pm 16.6$ & NS \\
\hline
\end{tabular}

$(26 \%)$ or an insufficiently filled maternal urinary bladder $(12 \%)$ relatively interfered with the cervical length investigation. Through the option of sagittal as well as transverse scans in 3D-mode, these anatomic difficulties did not present an obstacle to volumetry.

Aside from the acquisition of metric parameter data, ultrasound also allowed judgement of cervical configuration (fig. 2). Funneling of the internal os was found in $41 \%$ $(14 / 34)$, an opened cervical canal in $26 \%(9 / 34)$ and the amniotic sac protruding through the external os of the cervix was present in $9 \%(3 / 34)$. In $59 \%(20 / 34)$ of ultrasound scans, a closed cervix uteri (T-form) was recorded [18]. No single cervical configuration had relevant predictive value with regard to pregnancy duration. Finally, no cervical conformation was found to correlate with cervical length or volume (table 2).

Mean cervical length (2D), scanned in a (median) sagittal plane was $28.7 \pm 7.7 \mathrm{~mm}$. After reconstruction from 


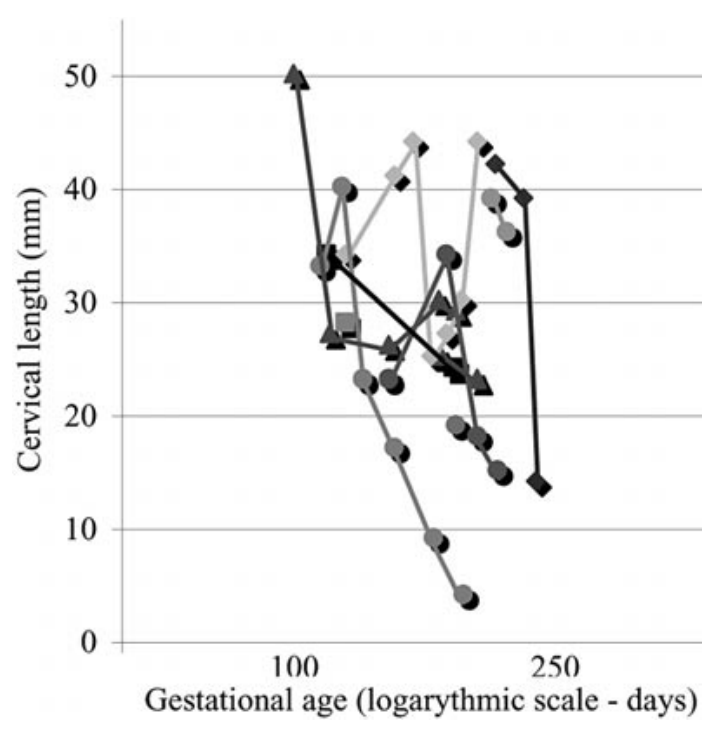

Fig. 4. Longitudinal course of cervical length corresponding to gestational age in the studied pregnancies (logarithmic scale).

the 3D-data, cervical length and configuration were reassessed and compared to the 'conventionally assessed' 2Dresults. Mean reconstructed cervical length was $32.2 \pm$ $10.9 \mathrm{~mm}$ and thus not significantly different from the conventional assessments (fig. 3). As expected, longitudinal analysis in individual higher order multiple pregnancies revealed a steady decrease of cervical length with gestational age (fig. 4).

Mean cervical volume was $30.0 \pm 16.0 \mathrm{~cm}^{3}$. With gestational age, the volume declined together with the 2Dlength measurements. This association is outlined in linear regression with a correlation coefficient, $r=0.83$ $(\mathrm{p}=0.01)$ (fig. 5). Detailed data concerning the sonographic examinations is described in table 3.

As mentioned above, Bishop's score did not correspond to preterm uterine contractions or the need for betamimethic therapy in the pregnancies studied. Furthermore none of the sonographic parameters - cervical conformation, length $(p=0.715)$ or volume $(p=0.469)$ significantly matched the pelvic score.

Multiparous women are more likely to develop cervical incompetence in a higher order multiple pregnancy. The influence of parity on the necessity of prenatal treatment, gestational age at delivery and sonographic surveillance parameters is evaluated in table 4 .
Table 3. Cervical sonography in the studied triplet and quadruplet pregnancies (mean, SD)

Number of sonographic cervical examinations Gestational age at initial cervical examination, days $3.4 \pm 2.1$

$176 \pm 28$ $12.3 \pm 7.6$ Time span between of cervical examinations, days $28.7 \pm 7.7$

$32.2 \pm 10.9$ Cervical length, conventional measurement, $\mathrm{mm}$ Reconstructed cervical length from 3D-scan, mm $30.0 \pm 16.0$

A subgroup analysis on cervical scans focused on the need for intravenous tocolysis (preterm labor, cervical incompetence) did not reveal significant differences: 3D-ultrasound length measurements at a mean of $34.1 \pm$ $11.4 \mathrm{~mm}$ in mothers with tocolysis are opposed to $24.5 \pm$ $2.1 \mathrm{~mm}$ in pregnancies without betamimethic treatment for preterm labor $(p=0.148)$. Cervical volumes, mean $33.4 \pm 16.2 \mathrm{~cm}^{3}$ could be compared to $16.5 \pm 3.5 \mathrm{~cm}^{3}$ respectively $(\mathrm{p}=0.192)$.

\section{Discussion}

Preterm birth remains the greatest single threat to survival and health of infants from a multiple pregnancy. Close clinical monitoring of cervical competence and premature labor is essential. Despite advances in perinatology, our ability to effectively predict preterm delivery is still lacking. Bed rest, hospitalization, antibiotics and tocolytics are regularly used to sustain the pregnancy when threatened by preterm labor, PPROM or cervical dilatation [18]. No single therapeutic initiative has been shown to reduce the incidence of preterm delivery effectively in the last years [3].

Among the higher order multiple pregnancies analyzed, cervical dilatation as indication for subsequent delivery was as frequent as $40 \%$. Therefore, identification of pregnancies at risk for cervical incompetence gains importance. Serial cervical assessments of women with multifetal gestation have revealed 'harmless' cervical changes that progress with gestational age. The process of cervical ripening can occur independently of uterine contractions, as it is known that both endocrine factors and the prostaglandin cascade can alter cervical consistency. However, preterm labor plays a major role in cervical effacement and dilatation [8].

Apart from palpation or direct inspection in the evaluation of the cervix, sonography is the standard monitoring 
Fig. 5. Linear correlation between cervical length (2D) and cervical volume (3D) measurements. Correlation coefficient: $r=0.83$.

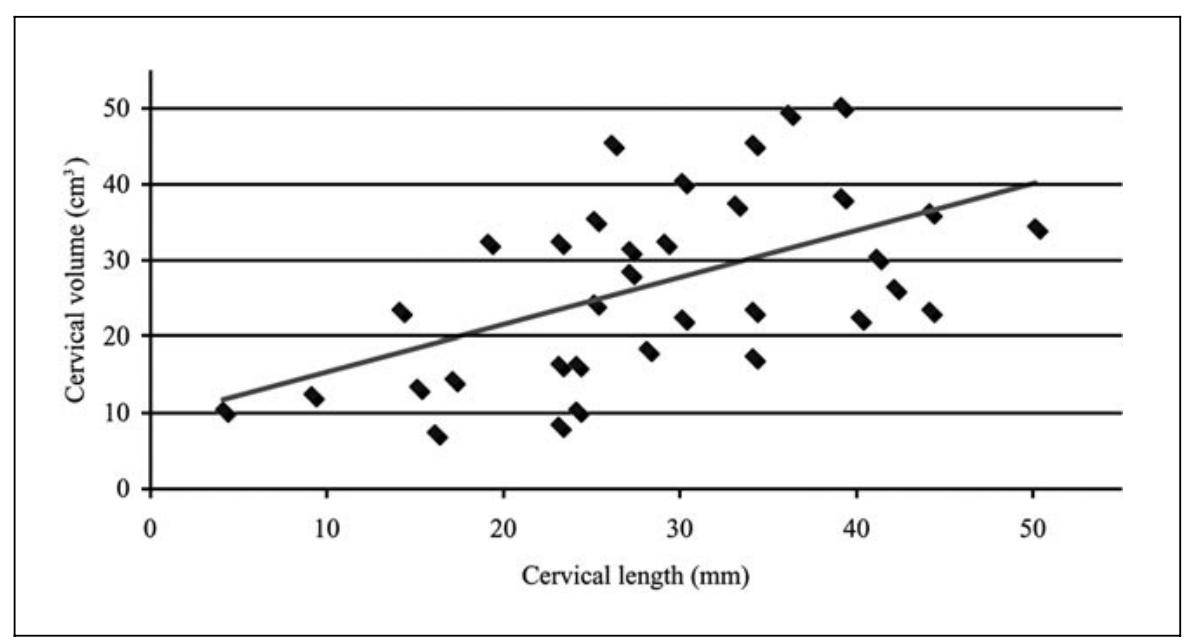

Table 4. Parity and cervical changes (mean, SD)

\begin{tabular}{lccc}
\hline & Primiparity & Multiparity & \\
\hline Cervical volume, $\mathrm{cm}^{3}$ & $33.5 \pm 16.0$ & $16.0 \pm 4.2$ & NS \\
Cervical length, mm & $30.3 \pm 7.8$ & $22.5 \pm 2.1$ & NS \\
Gestational age at hospitalization, days & $177 \pm 26$ & $172 \pm 21$ & NS \\
Tocolysis, \% & 87.5 & 50 & NS \\
Time span of tocolysis, days & $31.6 \pm 23.9$ & $6.0 \pm 8.5$ & NS \\
Gestational age at delivery, days & $215 \pm 21$ & $216 \pm 6$ & NS \\
\hline
\end{tabular}

cervical changes $[17,19]$. The introduction of 3D-ultrasound and cervical volumetry offers a new diagnostic option. The aim of sonographic measurements in multifetal gestation, however, cannot be the prediction of preterm birth in the literal sense, as all higher order multiples are generally delivered before 37 weeks. Instead, surveillance of treatment (bed rest, hospitalization, steroids, antibiotics, tocolytics) is the foremost diagnostic issue. Repeated palpation of the pelvis potentially promotes ascending infections, and thus may augment a risk it aims to control. Therefore, a reduction in vaginal manipulation without a loss of diagnostic information is a major advantage of transabdominal ultrasound. On the other hand there are studies that report even in the presence of ruptured membranes no increase in infectious morbidity after transvaginal ultrasound [20, 21]. It was the aim of this study to confirm the feasibility of cervical volume assessment in higher order multiples during the course of pregnancy, as well as to investigate its correlation compared to the established monitoring options. Do we achieve new information that warrants the increased tech- nical effort (3D-ultrasound) when compared to conventional (2D) length assessment?

In 10 pregnancies, clinical assessment (pelvic score) and sonography were compared at regular intervals. While it was possible to record 3D-scans in all patients and at all examinations, 2D-length measurements of the cervix were obtainable in all but 2 exams (94\%). In these 2 scans mentioned, 2D-length measurement of the cervix was anatomically obstructed by the presenting fetal part deep in the maternal pelvis. A relative impairment of the assessment of cervical conformation, length and volume as caused by an obstructing fetal head or an inadequately filled maternal urinary bladder was noted in over one third of ultrasound exams. This problem presented an obstacle in both 2D- and 3D-scans. We note that cervical operation appeared not to interfere with the ultrasound inspection. The quadruplet mother had undergone repeated cervical cerclage (2), yet the sonographic cervical assessment during the course of her pregnancy was not impaired. 
Serial cervical assessment in women with multifetal gestation revealed a progressive decrease of cervical volume which paralleled the decrease in cervical length. Volumetry correlated slightly closer to the sonographic gold standard - 2D-length measurement - than cervical length as reconstructed from 3D-scans. In women suffering from cervical incompetence, the relation between 3D- and 2Dresults was not altered. Aside from 2D metric changes, an alteration in the shape of the cervix is known to be associated with cervical incompetence [6]. Different authors stress the important difference between symptoms of cervical incompetence and preterm labor; the two can overlap because cervical dilatation caused by membrane herniation can induce uterine contractions. The sonographic evaluation of the cervix uteri assesses more than just cervical length, but also visualizes the shape of the internal os and cervical canal. These criteria are missed by palpation. Funneling of the internal os, an open cervical canal, or even a prolaps of amniotic sac through the external os can be obtained by conventional and 3D-assessment. In our study, these changes in cervical conformation showed no significant correlation to gestational age or prolongation of pregnancy. We were not able do identify diagnostic improvement in regard to cervical conformation when comparing volumetry to metric parameters. Also, no differences were found when parity was matched to sonographic data, need for hospitalization or gestational age at delivery. Our expectation that volumetry might unveil cervical changes associated with the need of intravenous tocolysis were also disappointed.

The data presented illustrates the methodical reproducibility of sonographic volume measurements of the cervix uteri in multifetal gestation. We had hoped that time-independent work-up of stored cervical volumes would allow more sophisticated data interpretation and thus more reliable clinical control. These expectations were not met. A potential explanation as to why volumetry did not improve our ability to monitor cervical changes is the challenge of the exact morphological differentiation of cervix and isthmus uteri. The technique of 3D-ultrasound has met limitations whenever applied in cervical assessment. We found particular limitations when, in higher gestational age, planes were obstructed through a fetal body or an insufficiently filled maternal urinary bladder. In our study, 3D-cervical scans were superior to $2 \mathrm{D}$-scans only in the cases when conventional measurements were technically unobtainable. Although cervical volumetry potentially extends our realm of methods to monitor the cervix in $6 \%$ of investigated cases, it did not enhance the overall information gained through the technically simple, conventional 2D-ultrasound.

\section{Conclusion}

Transabdominal ultrasound monitoring of the cervix is a well established and beneficial alternative to pelvic palpation in multifetal gestation. A fetal body deep in the pelvis can obstruct the conventional (2D) ultrasound assessment. Transverse volume scanning is the solution in these cases. Transabdominal 3D-volumetry as compared to 2D-cervical length assessment represents a reproducible instrument in all multiple pregnancies. Nevertheless, the technically more complex 3D-cervical visualization does not provide further metric or geometric information except under difficult scanning conditions in advanced gestational age. Although the number of pregnancies studied is still small we can conclude that cervical volumetry marks an effective extension in the surveillance of the cervix, avoiding palpation in higher order multiple pregnancies. Nevertheless, the employment of 3D-ultrasound needs to follow its appropriate indications.

\section{References}

1 Crowther CA: Prevention of preterm birth in multiple pregnancy. Baillières Clin Obstet Gynaecol 1998;12:67.

2 Strauss A, Hepp H: Höhergradige Mehrlinge perinatologische Herausforderung und Konsequenzen. Gynäkologe 1998;31:275.

3 Jewelewicz R: Incompetent cervix: Pathogenesis, diagnosis and treatment. Semin Perinatol 1991;15:156-161.

4 Michaels WH, Schreiber FR, Padgett RJ, Ager J, Pieper D: Ultrasound surveillance of the cervix in twin gestations: Management of cervical incompetence. Obstet Gynecol 1991;78:739744.
5 Neilson JP, Verkuyl DAA, Cowther CA, Bannerman $C$ : Preterm labor in twin pregnancies: Prediction by cervical assessment. Obstet Gynecol 1988;72:719-723.

6 Houlton MCC, Marivate M, Philpott RH: Factors associated with preterm labour and changes in the cervix before labour in twin pregnancy. Br J Obstet Gynaecol 1982;89:190.

7 Bishop EH: Pelvic scoring for elective induction. Obstet Gynecol 1964;24:266.
8 Newman RB, Godsey RK, Ellings JM, Campbell BA, Eller DP, Miller III MC: Quantification of cervical change: Relationship to preterm delivery in the multifetal gestation. Am J Obstet Gynecol 1991;165:264-269.

9 Iams JD, Goldenberg RL, Meis PJ, Mercer BM, Moawad A, Das A, Thom E, McNellis D, Cooper RL, Johnson F, Roberts JM: The length of the cervix and the risk of spontaneous premature delivery. New Engl J Med 1996;334: $567-572$. 
10 Romero R: The role of ultrasound in the prevention of preterm delivery. 10th World Congress on Ultrasound in Obstetrics and Gynecology 2000; Zagreb, Croatia.

11 Andersen HF, Nugent CE, Wantyn SD, Hayashi H: Prediction of risk for preterm delivery by ultrasonographic measurement of cervical length. Am J Obstet Gynecol 1990;163:859 867.

12 Ayers JWT, DeGrood RM, Crompton AA, Darclay M, Ansbacher R: Sonographic evaluation of cervical length in pregnancy: Diagnosis and management of preterm cervical effacement in patients at risk for premature delivery. Obstet Gynecol 1988;71:939.

13 Chou CY, Hsu KF, Wan ST, Huang SC, Tzeng CC, Huang KE: Accuracy of three-dimensional ultrasonography in volume estimation of cervical carcinoma. Gynecol Oncol 1997;66:89-93.
14 Gilja OH, Smiewoll AI, Thune N, Matre K, Hausken T, Odegaard S, Berstad A: In vivo comparison of 3D-ultrasonography and magnetic resonance imaging in volume estimation of human kidneys. Ultrasound Med Biol 1995, p 25.

15 Kyei-Mensah A, Zaidi J, Pittrof R, Shaker A, Campbell S, Tan SL: Transvaginal three-dimensional ultrasound: Accuracy of follicular volume measurements. Fertil Steril 1996;65: 371-376.

16 Lee A, Kratochwil A, Stümpflen I, Deutinger J, Bernaschek G: Fetal lung volume determination by three-dimensional ultrasonography. Am J Obstet Gynecol 1996;175:588-592.

17 Hoesli IM, Surbek DV, Tercanli S, Holzgreve $\mathrm{W}$ : Three-dimensional volume measurement of the cervix during pregnancy compared to conventional 2D-sonography. Int $\mathrm{J}$ Gynecol Obstet 1999;64:115.
18 Strauss A, Knitza R, Ott M, Genzel-Boroviczèny O, Versmold H, Hepp H: Geburtshilfliche Besonderheiten höhergradiger Mehrlinge Fluch oder mehrfacher Segen. Geburtsh Frauenheilk 1997;57:500.

19 Guzman ER, Benito C, Hanley M: Sonography in the evaluation of the cervix during pregnancy. Curr Op Obstet Gynecol 1996;8:99.

20 Carlan SJ, Richmond LB, O'Brien WF: Randomized trial of endovaginal ultrasound in preterm premature rupture of membranes. Obstet Gynecol 1997;89:458-461.

21 Rizzo G, Capponi A, Angelini E, Vlachopoulou A, Grassi C, Romanini C: The value of transvaginal ultrasonographic examination of the uterine cervix in predicting preterm delivery in patients with preterm premature rupture of membranes. Ultrasound Obstet Gynecol 1998; 11:23-29. 